\title{
Arrêté du 26 décembre 1997 fixant le contenu du dossier type prévu à l'article R.152-8-5 du code de la santé publique à produire à l'appui d'une demande d'autorisation de pratiquer une étude sur l'embryon in vitro.
}

Le secrétaire d'Etat à la santé.

Arrête :

Art. 1er.

- Les établissements publics de santé et les laboratoires d'analyses de biologie médicale désirant pratiquer une étude sur l'embryon in vitro définie à l'article R.152-8-1 du code de la santé publique doivent produire, à l'appui de leur demande, le dossier type spécifique mentionné à l'article R.152-8-5. dont le contenu est fixé en annexe du présent arrêté (1).

Art. 2.

- Le dossier fixé en annexe du présent arrêté doit être accompagné :

- pour chaque responsable proposé, d'un curriculum vitae ainsi que de la référence des travaux qu'il a effectués dans le domaine de l'étude demandée ;

- le document d'information prévu à l'article R.152-8-5 au vu duquel sera sollicité le consentement des deux membres du couple dont les embryons seront soumis à l'étude.

\section{Art. 3.}

- Le dossier type spécifique est à retirer auprès du secrétariat de la Commission nationale de médecine et de biologie de la reproduction et du diagnostic prénatal (direction générale de la santé, bureau SP2). 1, place de Fontenoy, 75007 Paris.

L'ensemble du dossier dûment rempli est retourné par le demandeur en trois exemplaires à l'adresse indiquée ci-dessus.

(1) L'arrêté accompagné de l'annexe sera publié intégralement au Bulletin officiel du ministère de l'emploi et de la solidarité $n^{\circ} 98-5$. disponible à la Direction des Journaux officiels. 26, rue Desaix. 75727 Paris Cedex 15, au prix de 40 F. 